\title{
Interaction between muscle and bone, and improving the effects of electrical muscle stimulation on amyotrophy and bone loss in a denervation rat model via sciatic neurectomy
}

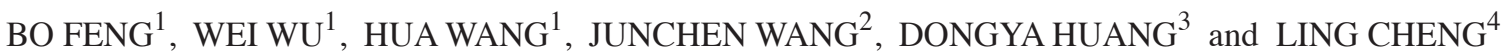 \\ Departments of ${ }^{1}$ Endocrinology, ${ }^{2}$ Pathology, ${ }^{3}$ Neurology and ${ }^{4}$ Traditional Chinese Medicine, \\ Shanghai East Hospital, Tongji University School of Medicine, Shanghai 200120, P.R. China
}

Received December 10, 2015; Accepted March 1, 2016

DOI: $10.3892 /$ br.2016.637

\begin{abstract}
The side-to-side difference in bone mineral content and soft tissue composition of extremities and their associations have been observed in patients with stroke and the results are inconsistent. The aim of the present study was to investigate the interaction between bone mineral content (BMC), lean mass (LM) and fat mass (FM) in the paretic extremities in patients following stroke and to determine the effectiveness of electrical muscle stimulation (EMS) following sciatic neurectomy (SN) in rats. BMC, LM and FM were measured by dual-energy X-ray absorptiometry in 61 hemiplegic patients following stroke. In the rat model study, groups of 10 Sprague-Dawley rats were divided into EMS and non-EMS subgroups. Myostatin expression and tetracycline interlabel width were measured. There were significant decreases in BMC, LM and FM in paretic limbs compared to non-paretic limbs. Compared to non-EMS, downregulated myostatin mRNA, and upregulated mechano growth factor (MGF) and insulin-like growth factor 1 (IGF-1) mRNA expression levels were observed in the EMS subgroup $(\mathrm{P}<0.05)$. In conclusion, muscle may have an important role in maintaining BMC. EMS-induced muscle contraction effectively downregulated myostatin mRNA, upregulated MGF and IGF-1 mRNA expression in muscle fiber, and mitigated amyotrophy and cortical bone loss from SN.
\end{abstract}

Correspondence to: Dr Bo Feng, Department of Endocrinology, Shanghai East Hospital, Tongji University School of Medicine, 150 Ji Mo Road, Shanghai 200120, P.R. China

E-mail: fengbo@medmail.com.cn

Abbreviations: BMC, bone mineral content; LM, lean mass; FM, fat mass; EMS, electrical muscle stimulation; MGF, mechano growth factor; $\mathrm{SN}$, sciatic neurectomy; SP, sham procedure; $\mathrm{BC}$, blank control; TC, tetracycline

Key words: amyotrophy, myostatin, growth factor, electronic muscle stimulation

\section{Introduction}

There is a higher risk of bone fracture in patients with stroke compared to the healthy controls. A significant reason for this is the decreased use of paretic extremities, which induced disuse amyotrophy and bone loss (1). In a few previous studies the associations of bone mineral content (BMC) and soft tissue composition of the extremities were observed in patients with stroke and the results were inconsistent $(2,3)$. One of the reasons was that different methodologies for rehabilitation were used.

There is a hypothesis that skeletal muscle should not be only treated as a locomotorium, but also as an endocrine organ $(4,5)$. Increasing research over the past 20 years has demonstrated that muscle is a source of myokines that can influence muscle and bone growth in positive or negative ways. Myostatin, insulin-like growth factor 1 (IGF-1) and mechano growth factor (MGF) are three significant myokines, which are produced, expressed and released by muscle fibers following external stimulation, and have their own roles in mediating bone and muscle metabolism by exerting either paracrine or endocrine effects (6-9).

The present study was divided to two sections; the clinical research and animal experiment. The clinical research was designed to investigate the association between BMC, lean mass (LM) and fat mass (FM) in the hemiplegic extremities in patients following a stroke, while the animal experiment was designed to determine the effectiveness of electrical muscle stimulation (EMS) to the hindlimbs following sciatic neurectomy ( $\mathrm{SN}$ ) in attenuating disuse amyotrophy and cortical bone loss in Sprague-Dawley female rats by regulating the myostatin, IGF-1, and MGF mRNA or protein expression in muscle fiber and bone tissue.

\section{Materials and methods}

\section{Clinical research}

Subjects. A total of 61 hemiplegic patients subsequent to experiencing a stroke [35 men, mean age of 64.3 years, mean body mass index (BMI) of $23.9 \mathrm{~kg} / \mathrm{m}^{2} ; 26$ women, mean age of 65.8 years, mean BMI of $25.7 \mathrm{~kg} / \mathrm{m}^{2}$ ) were recruited on a volunteer basis. All the subjects had to fulfill the following 
inclusion criteria: i) Experienced one single stroke only, ii) post-stroke duration of $\geq 1$ year, and iii) were independent in ambulation with or without an assistive device. Potential subjects were excluded if they: i) Had other serious diseases in addition to stroke that affect bone metabolism, ii) had any metal implants in extremities, iii) were currently prescribed medications that affect bone metabolism, and iv) had unstable cardiovascular disease.

Dual-energy X-ray absorptiometry (DEXA). Each subject underwent a total body scan using DEXA (Lunar-DPX IQ; GE Lunar Corp., Chicago, IL, USA). All the scans were performed by the same technician using standard procedures, following the manufacturer's protocol. The BMC $(\mathrm{g}), \mathrm{LM}(\mathrm{g})$ and FM $(\mathrm{g})$ in each extremity were determined by the region of interest program.

\section{Animal experiment}

Animal and experimental design. A total of 30 female specific pathogen-free Sprague-Dawley rats were obtained from Sino-British Sippr/BK Lab Animal Ltd., Co. [Shanghai, China; license no. SCXK (HU) 2008-0016] weighing $200 \mathrm{~g}$ ( 6 weeks of age) and allowed to acclimate to their surroundings for 1 week prior to the initiation of the study. All the rats were housed in the Experimental Animal Center of Shanghai University of Traditional Chinese Medicine (Shanghai, China). The animal room was environmentally controlled at $28 \pm 1^{\circ} \mathrm{C}$ and $60 \%$ humidity with a 12:12-h light-dark cycle.

Three experimental groups were studied: i) Blank control (BC, $n=10)$, ii) sham procedure (SP, $n=10)$, and iii) animals that underwent surgery and were subjected to right-side SN $(\mathrm{n}=10)$ under anesthesia pentobarbital sodium $(0.1 \mathrm{ml} / 100 \mathrm{~g}$ body weight). Rats were housed with normal cage activity and allowed free access to standard lab chow and tap water. Each group was divided into 2 subgroups: EMS and non-EMS. EMS was performed on the rats in the EMS subgroup. After 9 weeks all the rats were sacrificed.

At the time of sacrifice the soleus, gastrocnemius, medial vastus and tibial anterior muscle were excised, and the wet weights were recorded. Additionally, the tibias were removed and weighed, cleaned of soft tissue and stored in $75 \%$ ethanol at room temperature for cortical histomorphometry of the diaphysis region. The femurs were stored in ethylenediaminetetraacetic acid (5.5 g:100 ml 10\% formalin) at room temperature for detecting myostatin protein expression with an immunohistochemical method.

EMS. EMS was performed in rats with limbs fixed by threads. The stainless steel needles were inserted percutaneously into the tibial anterior muscle, at a depth of $7 \mathrm{~mm}$ at the knee region of rats. The stimulation was applied using a G9805 stimulator (Medical Electronic Instrument Factory, Shanghai, China) with $1.0 \mathrm{~mA}$ and a duration of $1.0 \mathrm{msec}$ at 3 pulses. Rats in the EMS subgroups underwent EMS for a period of $30 \mathrm{~min}$ at a time, once a day for 5 days a week, for a duration of 9 weeks. The limbs of the rats in the non-EMS subgroup were fixed and needles were placed into the same point without performing the stimulation.

Reverse transcription-quantitative polymerase chain reaction (RT-qPCR). RNA extraction was performed according to the
RNAiso Plus directions (D9108A; Takara, Tokyo, Japan) with a few modifications. Subsequently, the integrity of the total RNA was confirmed by determination of the optical density (OD) at 260 and $280 \mathrm{~nm}$ under ultraviolet light (Shimadzu, Kyoto, Japan). Only those samples with OD260/OD280 $\geq 1.7$ were used for RT. RT reactions were carried out on 1-2 $\mu \mathrm{g}$ of sample in accordance with the PrimeScript RT reagent kit (DRR037A; Takara) using a DNA Engine Peltier Thermal Cycle machine (Bio-Rad, Hercules, CA, USA). Samples were analyzed for myostatin, IGF-1, and MGF mRNA levels by RT-qPCR using the Real-Time 7500 system (Applied Biosystems, Thermo Fisher Scientific, Inc., Waltham, MA, USA) using SYBR Premix Ex Taq kit (Takara code: DRR041A). The sequences of primers used for PCR were as follows: GAPDH-forward (F), GACAACTTT GGCATCGTGGA and reverse (R), ATGCAGGGATGATGT TCTGG; myostatin-F, AGAATGGGCATGATCTTGCTG TAAC and R, CATCACAGTCAAGCCCAAAGTCTC; MGF-F, GCTTGCTCACCTTTACCAGC and R, AAATGT ACTTCСTTTCCTTCTC; and IGF-F, TTCAGTTCGTGT GTGGACCAAG; R, GATCACAGCTCCGGAAGCAA. All the primer sequences were designed according to gene bank and synthesized by Takara.

Histology and immunohistochemistry. Vastus medialis and femurs, harvested from rats anesthetized by $3 \%$ pentobarbital sodium $(0.1 \mathrm{ml} / 100 \mathrm{~g}$ body weight), were embedded in paraffin (following fixation in $4 \%$ formalin). The paraffin blocks were separated at $5 \mu \mathrm{m}$ and vastus medialis atrophy was determined through histomorphometry following hematoxylin and eosin (H\&E) staining. After deparaffinization and rehydration in ascending grades of alcohol, antigen retrieval was performed in citrate buffer $(\mathrm{pH} 6.0)$ at $93-98^{\circ} \mathrm{C}$ for $6 \mathrm{~min}$ in the microwave. The slides were blocked in $10 \%$ fetal bovine serum (Sigma-Aldrich, St. Louis, MO, USA) for $15 \mathrm{~min}$. The sections of vestus medialis and femur were stained with rabbit antihuman yostatin (sc-28910, 1:100, Santa Cruz Biotechnology, Inc., Dallas, TX, USA) for $2 \mathrm{~h}$ at room temperature and staining was revealed with a biotinylated goat anti-rabbit secondary antibody (sc-2018, 1:200, Santa Cruz Biotechnology, Inc.) for $1 \mathrm{~h}$ at room temperature. Endogenous peroxidase activity was blocked by $3 \% \mathrm{H}_{2} \mathrm{O}_{2}$ and slides were counterstained with $\mathrm{H} \& \mathrm{E}$.

Histomorphometric analysis. Tetracycline (TC; Xinhua Pharmaceutical Co., Ltd., Shandong, China) gavage $(100 \mathrm{mg} / \mathrm{kg}$ of body mass) was administered to rats on day 1 of EMS and the day prior to sacrificing to label the mineralizing bone formation for histomorphometric analysis.

Undemineralized distal tibiae were subjected to serial dehydration. Serial cross sections (200-250 $\mu \mathrm{m})$ of midshaft bone were separated starting at $3 \mathrm{~mm}$ with a Leica SP1600 slicing machine (Leica, Wetzlar, Germany). Sections were handground to reduce the thickness $(<100 \mu \mathrm{m})$ prior to mounting on glass slides. The histomorphometric analyses were performed using the Olympus microscope (Olympus, Tokyo, Japan). Measures of labeled surfaces and interlabel widths were obtained (magnification, x200) of $\leq 3$ slides per tibiae. The cortical grow rates were calculated by dividing the average interlabel width by the time between the two times of labeling (63 days). 
Table I. Comparison between the paretic and non-paretic extremities.

\begin{tabular}{lccc}
\hline Variable & $\begin{array}{c}\text { Non-paretic } \\
\text { extremity }\end{array}$ & $\begin{array}{c}\text { Paretic } \\
\text { extremity }\end{array}$ & $\begin{array}{c}\text { P-value } \\
\text { (paired t-tests) }\end{array}$ \\
\hline Upper & & & \\
extremity & & & \\
BMC, g & $166.5 \pm 56.8$ & $138.9 \pm 54.8$ & $<0.01$ \\
FM,g & $1677.3 \pm 852.5$ & $1437.4 \pm 755.2$ & $<0.01$ \\
LM,g & $2994.5 \pm 1003.9$ & $2515.9 \pm 812.1$ & $<0.01$ \\
LM/FM & $2.35 \pm 1.4$ & $2.4 \pm 2.1$ & $>0.05$ \\
Lower & & & \\
extremity & & & $<0.01$ \\
BMC, g & $434.8 \pm 138.1$ & $398.0 \pm 142.5$ & $<0.01$ \\
FM, g & $2393.2 \pm 884.2$ & $2237.1 \pm 872.7$ & $<0.01$ \\
LM,g & $6541.3 \pm 1633.3$ & $6090.6 \pm 1693.8$ & $>0.05$ \\
LM/FM & $3.2 \pm 1.7$ & $3.3 \pm 1.8$ & \\
\hline
\end{tabular}

BMC, bone mineral content; FM, fat mass; LM, lean mass.

Table II. Wet weight of muscle and bone from the left and right hindlimbs of the different treatment groups.

Group Sample Left hindlimb,g Right hindlimb,g P-value

\begin{tabular}{lllll}
\hline BC & Vastus & $2.29 \pm 0.31$ & $2.41 \pm 0.42$ & 0.33 \\
& medialis & & & \\
& Soleus & $2.05 \pm 0.20$ & $2.05 \pm 0.26$ & 0.90 \\
& TA & $1.11 \pm 0.43$ & $1.17 \pm 0.48$ & 0.45 \\
& Femur & $0.97 \pm 0.15$ & $1.03 \pm 0.14$ & 0.27 \\
& Tibia & $0.84 \pm 0.21$ & $0.80 \pm 0.07$ & 0.52 \\
SP & Vastus & $2.39 \pm 0.28$ & $2.26 \pm 0.33$ & 0.27 \\
& medialis & & & \\
& Soleus & $2.17 \pm 0.20$ & $2.07 \pm 0.24$ & 0.37 \\
& TA & $1.02 \pm 0.11$ & $0.97 \pm 0.18$ & 0.38 \\
& Femur & $1.04 \pm 0.13$ & $1.05 \pm 0.14$ & 0.46 \\
& Tibia & $0.77 \pm 0.11$ & $0.76 \pm 0.10$ & 0.71 \\
SN $\quad$ Vastus & $2.04 \pm 0.23$ & $1.86 \pm 0.30$ & 0.03 \\
& medialis & & & \\
& Soleus & $1.85 \pm 0.19$ & $0.59 \pm 0.47$ & 0.01 \\
& TA & $0.84 \pm 0.11$ & $0.35 \pm 0.15$ & 0.01 \\
& Femur & $1.04 \pm 0.11$ & $0.89 \pm 0.11$ & 0.01 \\
& Tibia & $0.74 \pm 0.06$ & $0.68 \pm 0.06$ & 0.01 \\
\hline
\end{tabular}

BC, blank control; SP, sham procedure; SN, sciatic neurectomy; TA, tibialis anterior.

Statistical analysis. All data are expressed as mean \pm standard deviation and evaluated using the statistical package SPSS 13.0 (SPSS, Inc., Chicago, IL, USA). Paired t-tests were used to examine whether there were side-to-side differences in BMC, LM and FM. Pearson correlations were used for nominal data that were normally distributed: BMC, LM, and FM. Bone, muscle wet weight at sacrifice, myostatin, IGF-1 and
MGF mRNA expression levels and TC interlabel width were analyzed using paired t-tests to determine whether there were significant differences between operated and contralateral hindlimbs. The unpaired t-test was used to compare the group and subgroup differences. For all data, $\mathrm{P}<0.05$ was considered to indicate a statistically significant difference.

Ethical statement. All patients participating in the present study provided written informed consent. The protocols for the clinical research and animal experiments were approved by all hospital ethics committees.

\section{Results}

Clinical investigation

Comparison of bone mineral levels and soft tissues composition between the extremities. Bone mineral levels and soft tissue composition were significantly different between paretic and non-paretic extremities (Table I). The paretic extremities had a significantly lower BMC, FM and LM compared to non-paretic extremities. No significant differences were identified in the LM/FM ratio between the paretic and non-paretic extremities.

BMC loss and muscle atrophy. There were significant correlations between BMC and LM, but not FM in the non-paretic upper $(r=0.754, \mathrm{P}<0.01)$ and lower $(\mathrm{r}=0.877, \mathrm{P}<0.01)$ extremities. However there were significant correlations between BMC and LM $(r=0.808, \mathrm{P}<0.01)$ and FM $(r=-0.305, \mathrm{P}<0.05)$ in the paretic upper extremity and only between $\mathrm{BMC}$ and $\mathrm{LM}(\mathrm{r}=0.884, \mathrm{P}<0.01)$ in paretic lower extremity. A decrease in BMC was significantly correlated with LM loss (upper: $\mathrm{r}=0.808$; lower: $\mathrm{r}=0.884 ; \mathrm{P}<0.01)$ in the paretic extremities, while there was no evidence of BMC loss being correlated with FM loss. The multiple regression analysis showed that extremity LM loss was a strong predictor of paretic extremity BMC loss (upper: $\beta=0.702, F=16.244, P<0.01$; lower: $\beta=0.836$, $\mathrm{F}=47.374, \mathrm{P}<0.01)$.

\section{Rat model experiments}

Changing trend of body weight. The mean weight increase of the $\mathrm{BC}, \mathrm{SP}$ and SN groups from the first to the last week was 28.33, 42.32 and $20.24 \%$. The EMS group weight only increased by $23.65 \%$, while the non-EMS group had a growth of $27.58 \%$.

Influence of SN on the wet weight of muscle and bones. There were no differences of vastus medials, soleus, tibialis anterior (TA) muscle, femur and tibia wet weight between the right and left hindlimbs in the $\mathrm{BC}$ and SP groups $(\mathrm{P}>0.05)$. The SN group showed a significant loss of vastus medials, soleus, TA muscle, tibia and femur wet weight in the surgically operated hindlimbs compared to contralateral limbs $(\mathrm{P}<0.01)$ (Table II). Compared to the BC group, no difference was identified in the SP group in muscle or bone wet weight in the left or right hindlimbs $(\mathrm{P}>0.05)$, while the $\mathrm{SN}$ group had a significant wet weight loss in the surgically operated hindlimbs $(\mathrm{P}<0.01)$.

Histology and immunohistochemistry. In sections from vastus medialis with $H \& E$ staining, muscle fiber atrophy was 
Table III. Relative expression levels of MSTN, MGF and IGF-1 mRNA in muscle fibers of the different treatment groups.

\begin{tabular}{|c|c|c|c|c|c|c|}
\hline Gene & BC+Non-EMS & $\mathrm{BC}+\mathrm{EMS}$ & SP+Non-EMS & SP+EMS & SN+Non-EMS & $\mathrm{SN}+\mathrm{EMS}$ \\
\hline MSTN & $1.00 \pm 0.00$ & $0.59 \pm 0.14$ & $0.80 \pm 0.17$ & $0.48 \pm 0.31$ & $3.32 \pm 1.40$ & $1.45 \pm 0.81$ \\
\hline MGF & $1.00 \pm 0.00$ & $1.35 \pm 0.56$ & $1.18 \pm 0.28$ & $1.66 \pm 0.75$ & $1.02 \pm 0.58$ & $2.76 \pm 1.00$ \\
\hline IGF-1 & $1.00 \pm 0.00$ & $1.34 \pm 0.39$ & $1.05 \pm 0.25$ & $1.74 \pm 1.16$ & $1.32 \pm 0.52$ & $2.71 \pm 1.36$ \\
\hline
\end{tabular}

MSTN, myostatin; MGF, mechano growth factor; IGF-1, insulin-like growth factor 1; BC, blank control; SP, sham procedure; SN, sciatic neurectomy; EMS, electrical muscle stimulation.

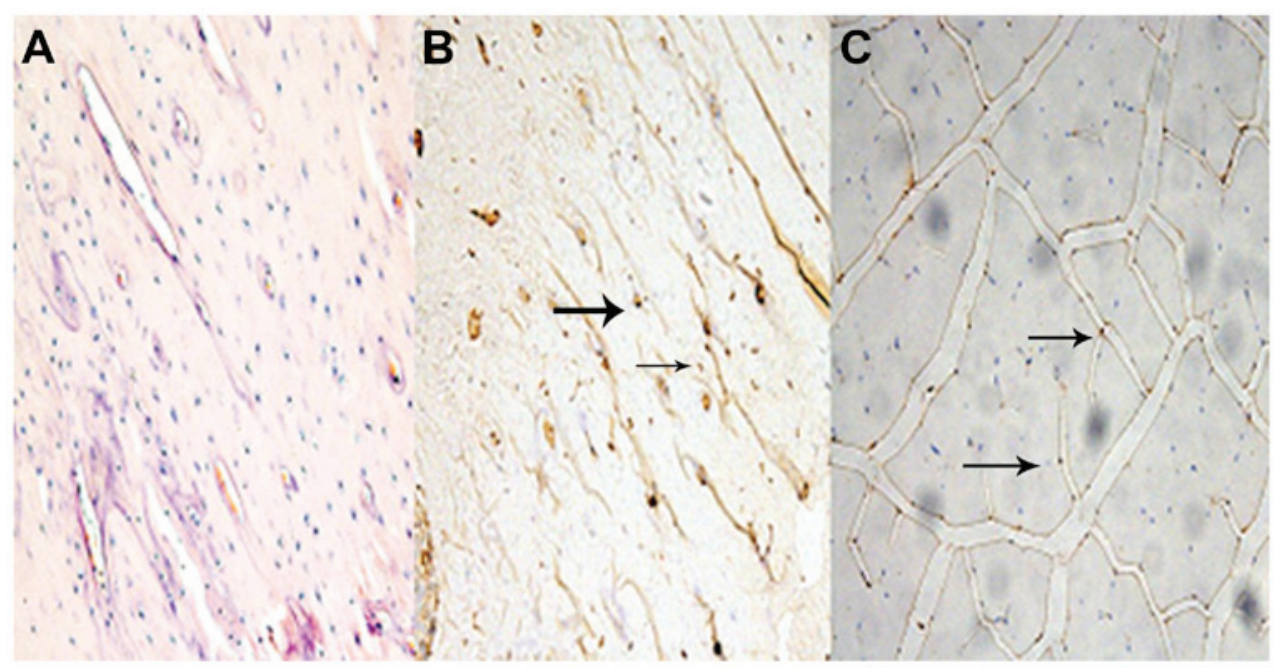

Figure 1. Immunohistochemical appearance of myostatin protein expression in femur tissue and vastus medialis muscle. (A) Femur tissue in the BC group; (B) femur tissue in the SN group; and (C) vastus medialis muscle in the SN group. All images are magnification, x200. Arrows show target myostatin protein expression. SN, sciatic neurectomy; BC, blank control.

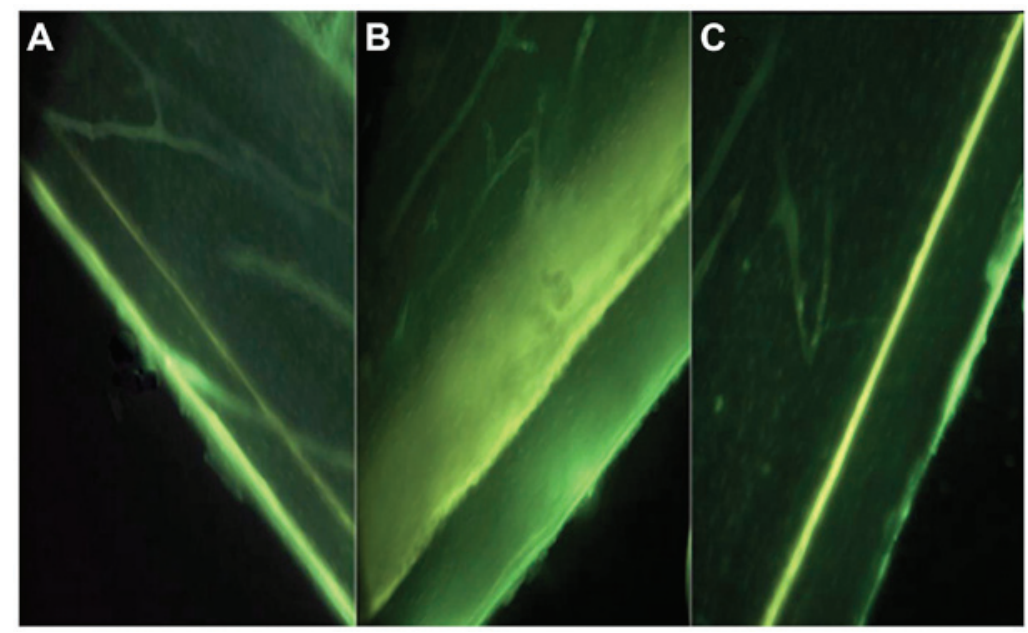

Figure 2. Visual depiction (magnification, x200) of TC labeling on the periosteal surface of cortical bone at the tibia diaphysis. (A) SN+non-EMS; (B) SN+EMS; and (C) BC. Of note is the smaller flurochrome labeling width in the SN+non-EMS group. SN+EMS and BC have similar large interlabel width. SN, sciatic neurectomy; EMS, electrical muscle stimulation; BC, blank control; TC, tetracycline.

observed in the SN group. All the groups maintained the polygonal muscle fiber shape.

Fig. 1 represents the sections of myostatin protein expression in femur bone tissue and medial vastus muscle fiber. Relative to the $\mathrm{BC}$ group, myostatin protein expression was observed in the bone and muscle tissue in the SN group.
Myostatin, MGF and IGF-1 mRNA expression levels. No differences were observed for the myostatin, MGF and IGF-1 mRNA expression levels between the SP and BC group $(\mathrm{P}>0.05)$. The $\mathrm{SN}$ group showed a significant upregulated myostatin mRNA expression level, while MGF and IGF-1 mRNA remained the same level compared to the BC group. 
Myostatin mRNA expression level was significantly decreased in the BC+EMS and SN+EMS subgroups, while there was a significant increase in the trend in MGF and IGF-1 mRNA expression had a significant increase trend compared to Non-EMS subgroup $(\mathrm{P}<0.05)$. However, no significant differences were observed in the SP+EMS and SP+non-EMS group (P>0.05) (Table III).

EMS increases bone formation on cortical surfaces. No significant differences were observed in the TC interlabel width between BC $(56.90 \pm 10.21 \mu \mathrm{m})$ and SP $(57.00 \pm 9.90 \mu \mathrm{m})$ groups $(\mathrm{P}>0.05)$. The $\mathrm{SN}$ group showed an evident decrease in TC interlabel width $(43.33 \pm 16.33$ vs. $57.50 \pm 17.47 \mu \mathrm{m})$ and cortical growth speed $(0.69 \pm 0.26$ vs. $0.91 \pm 0.28 \mu \mathrm{m} /$ day $)$ in the surgically operated hindlimbs compared to the contralateral hindlimbs, but was not significantly different $(\mathrm{P}>0.05)$. Animals in the SN group were exposed to EMS (SN+EMS), and this subgroup exhibited a relatively larger TC interlabel width and faster cortical growth speed ( $\mathrm{P}>0.05$ ) (Table IV and Fig. 2).

\section{Discussion}

Disuse muscle atrophy is observed in the paretic extremities in a number of patients following stroke, which correlates a higher risk of bone fractures. Reduced motor skills, failure to keep balance and osteoporosis may lead to this. There have been several studies on BMC and soft tissue changes in hemiplegic patients following stroke. BMC and LM were significantly decreased in paretic extremities compared to non-paretic extremities, and BMC correlated strongly with LM (1-3). The results of the present study supported these opinions. In addition, BMC was strongly correlated with LM in paretic and non-paretic extremities, and BMC loss was strongly correlated with LM loss, but not FM in paretic extremities of patients following stroke. The LM was an important predictor of BMC, indicating that muscle has a key role in maintaining BMC of extremities (1). Therefore, we may suppose that exercise could prevent bone loss and increase BMC through muscle stretching.

In order to investigate the molecular mechanism and discuss whether physical therapy could prevent LM and bone loss, a rat model of SN to the hindlimbs was established to assess the association of BMC loss with LM loss and the feasibility of EMS for attenuating cortical bone loss and muscle atrophy. The present study showed that rats with SN had a lower rate of weight increase compared to the other two groups and there was significant reduction in muscle and bone wet weight in the surgically operated hindlimbs compared to the non-operated sides, which may indicate that reduced muscle contraction due to muscle atrophy was associated with the cause of bone loss. As bone formation was stimulated by muscle forces, muscle strength may be a key factor affecting bone mineralization. EMS is a non-drug therapy. Wei et al (10) reported that loading by stimulation-induced muscle contraction at high frequencies was beneficial for the maintenance of bone growth or the prevention of mineral loss, or both, during hindlimb suspension in rats. In the present study, TC was used for dual-label mineralizing bone formation and bone growth. EMS increased bone growth rate, which suggests that EMS could prevent bone mineral loss due to SN through stimulating muscle contraction.
Table IV. TC interlabel width and growth speed on the tibia cortical surface of the different subgroup treatments.

\begin{tabular}{lllc}
\hline Groups & Tibia & $\begin{array}{c}\text { Interlabel } \\
\text { width, } \mu \mathrm{m}\end{array}$ & $\begin{array}{c}\text { Growth speed, } \\
\mu \mathrm{m} / \text { day }\end{array}$ \\
\hline BC+non-EMS & Right & $48.75 \pm 12.37$ & $0.77 \pm 0.20$ \\
BC+EMS & Right & $62.23 \pm 4.61$ & $0.99 \pm 0.07$ \\
SP+non-EMS & Left & $50.00 \pm 3.54$ & $0.79 \pm 0.06$ \\
& Right & $54.17 \pm 6.29$ & $0.52 \pm 0.48$ \\
SP+EMS & Left & $61.67 \pm 10.40$ & $0.98 \pm 0.17$ \\
& Right & $48.50 \pm 6.26$ & $0.46 \pm 0.43$ \\
SN+non-EMS & Left & $51.67 \pm 11.27$ & $0.82 \pm 0.18$ \\
& Right & $37.50 \pm 5.00$ & $0.36 \pm 0.33$ \\
SN+EMS & Left & $63.33 \pm 23.09$ & $1.01 \pm 0.37$ \\
& Right & $49.17 \pm 23.23$ & $0.78 \pm 0.37$ \\
\hline
\end{tabular}

TC, tetracycline; BC, blank control; SP, sham procedure; SN, sciatic neurectomy; EMS, electrical muscle stimulation.

The interaction between muscle and bone remains to be elucidated, and it was shown that muscle is a potential endocrine organ that could synthesis and secrete several cytokines to affect bone metabolism (5). Myostatin as an important myokine, formerly known as growth and differentiation factor 8 , a member of transforming growth factor- $\beta$ superfamily, is an important negative regulator of skeletal muscle mass (11). The data reported in the present study showed a significant upregulated myostatin mRNA level in gastrocnemius muscle fibers of surgically operated hindlimbs. Muscle without neurotrophy resulted in myostatin mRNA increase, which led to the inhibition of myocyte proliferation and muscle atrophy. However, following the administration of SN rat EMS, myostatin mRNA expression showed a clear decrease. This result supported the view that myostatin downregulation was in response to EMS, which was in line with other studies that showed resistance-type muscle loading could decrease myostatin mRNA expression levels (11). Furthermore, in the present study it was shown that myostatin protein was not only expressed in muscle tissue but also bone tissue, and changes in mRNA were indicative of changes in the protein levels. Considering the inhibiting effect of myostatin on muscle growth and bone growth (6-8), this finding could help to explain the superior effect of EMS on attenuating muscle atrophy and bone loss. As shown in the data presented, the SN+EMS subgroup showed larger TC interlabel width and faster cortical growth speed compared to the SN+non-EMS subgroup. Therefore, we may speculate that the faster growth of cortical bone is associated with a decrease of myostatin mRNA expression.

IGF-1 is synthesized by almost all tissues and is an important mediator of cell growth, differentiation, and transformation. IGF-1 is the product of the IGF-1 gene and it is known that the IGF-1 gene has several different splice variants that have different functions. These variants in rodents include one that is the same as the main systemic IGF-1Ea produced by liver and another which has been termed MGF (IGF-1Eb), 
and its mRNA is only detected in muscle following mechanical stimulation. From its sequence, MGF is derived from the IGF-1 gene by alternative splicing and has different 3 ' exons to IGF-1Ea, it has a 52 base-pair insert in rodents within the $\mathrm{E}$ domain of exon 5, which results in a different reading frame (9). Hill and Goldspink (12) reported that following mechanical stimulation or muscle damage, the IGF-1 gene was first spliced to produce MGF and later produced the more common IGF-1Ea transcript. MGF can induce satellite cell activation as well as protein synthesis. A mammalian expression plasmid containing the MGF cDNA sequence was delivered to the hindlimb muscles of mice, which resulted in a significant improvement in hindlimb muscle strength, and an increase in motor unit and motor neuron survival (13). No significant differences in IGF-1 and MGF mRNA expression levels in the $\mathrm{BC}, \mathrm{SP}$ and SN groups were observed in the present study. However, MGF and IGF-1 mRNA level presented a significant increase in the EMS subgroup compared to the non-EMS subgroup. This result demonstrated that EMS could upregulate MGF and IGF-1 mRNA levels in muscle fibers, which was consistent with other studies. Hameed et al (14) reported that high resistance exercise resulted in a significant increase (864\%) in MGF mRNA in young patients. Bamman et al (15) reported a $62 \%$ increase in IGF-1 mRNA level in human muscle $48 \mathrm{~h}$ after a single bout of eccentric resistance-type exercise. The present study showed an increase of MGF mRNA following EMS; however, the magnitude was not as high as other studies (16). It is possible that MGF is just a transient and activity-sensitive local growth factor with a short half life. In the present study, MGF mRNA in gastrocnemius muscle was detected $24 \mathrm{~h}$ later after the last EMS, and it is possible that the majority of MGF had already degraded. Considering the positive effect of MGF and IGF-1 on muscle growth, we may speculate that EMS could benefit in preventing muscle atrophy.

Certain limitations merit discussion. First, in the clinical research there was a relatively small sample size. Second, in the rat model experiments, BMD levels were not determined and ultrastructure of the bone was not observed by micro-computed tomography.

In conclusion, a decrease of BMC in paretic limbs correlated significantly with a decrease of LM, but not with FM, in patients following stroke. EMS mitigated disuse amyotrophy and bone loss from SN, associated with downregulating myostatin expression and increasing IGF-1 and MGF in muscle fiber, and enhancing bone formation.

\section{Acknowledgements}

The present study was funded by the Ministry of Civil Affairs China [grant no. (2007)18-1-09].

\section{References}

1. Pang MYC and Eng JJ: Muscle strength is a determinant of bone mineral content in the hemiparetic upper extremity: Implications for stroke rehabilitation. Bone 37: 103-111, 2005.

2. Iwamoto J, Takeda T and Ichimura S: Relationships between physical activity and metacarpal cortical bone mass and bone resorption in hemiplegic patients. J Orthop Sci 6: 227-233, 2001.

3. Sahin L, Ozoran K, Gündüz OH, Uçan H and Yücel M: Bone mineral density in patients with stroke. Am J Phys Med Rehabil 80: 592-596, 2001.

4. Nielsen S and Pedersen BK: Skeletal muscle as an immunogenic organ. Curr Opin Pharmacol 8: 346-351, 2008.

5. Engler D: Hypothesis: Musculin is a hormone secreted by skeletal muscle, the body's largest endocrine organ. Evidence for actions on the endocrine pancreas to restrain the beta-cell mass and to inhibit insulin secretion and on the hypothalamus to co-ordinate the neuroendocrine and appetite responses to exercise. Acta Biomed 78 (Suppl 1): 156-206, 2007.

6. Ma K, Mallidis C, Bhasin S, Mahabadi V, Artaza J, et al: Glucocorticoid-induced skeletal muscle atrophy is associated with upregulation of myostatin gene expression. Am J Physiol Endocrinol Metab 285: E363-E371, 2003.

7. Elkasrawy MN and Hamrick MW: Myostatin (GDF-8) as a key factor linking muscle mass and bone structure. J Musculoskelet Neuronal Interact 10: 56-63, 2010.

8. Hamrick MW, Samaddar T, Pennington C and McCormick J: Increased muscle mass with myostatin deficiency improves gains in bone strength with exercise. J Bone Miner Res 21: 477-483, 2006.

9. West CA, Arnett TR and Farrow SM: Expression of insulin-like growth factor I (IGF-I) mRNA variants in rat bone. Bone 19: 41-46, 1996.

10. Wei CN, Ohira Y, Tanaka T, Yonemitsu H and Ueda A: Does electrical stimulation of the sciatic nerve prevent suspension-induced changes in rat hindlimb bones? Jpn J Physiol 48: 33-37, 1998.

11. Roth SM, Martel GF, Ferrell RE, Metter EJ, Hurley BF and Rogers MA: Myostatin gene expression is reduced in humans with heavy-resistance strength training: A brief communication. Exp Biol Med (Maywood) 228: 706-709, 2003.

12. Hill $\mathrm{M}$ and Goldspink G: Expression and splicing of the insulin-like growth factor gene in rodent muscle is associated with muscle satellite (stem) cell activation following local tissue damage. J Physiol 549: 409-418, 2003.

13. Riddoch-Contreras J, Yang SY, Dick JR, Goldspink G, Orrell RW and Greensmith L: Mechano-growth factor, an IGF-I splice variant, rescues motoneurons and improves muscle function in SOD1(G93A) mice. Exp Neurol 215: 281-289, 2009.

14. Hameed M, Orrell RW, Cobbold M, et al: Expression of IGF-I splice variants in young and old human skeletal muscle after high resistance exercise. J Physiol 547: 247-254, 2003.

15. Bamman MM, Shipp JR, Jiang J, Gower BA, Hunter GR, Goodman A, et al: Mechanical load increases muscle IGF-I and androgen receptor mRNA concentrations in humans. Am J Physiol Endocrinol Metab 280: E383-E390, 2001.

16. Owino V, Yang SY and Goldspink G: Age-related loss of skeletal muscle function and the inability to express the autocrine form of insulin-like growth factor-1 (MGF) in response to mechanical overload. FEBS Lett 505: 259-263, 2001. 